\title{
The diversity, composition, and metabolic pathways of archaea in pigs
}

\section{Feilong Deng}

Foshan University

\section{Yushan Li}

Foshan University

Xiaoyuan Wei

University of Arkansas

Yingping Xiao

Zhejiang Academy of Agricultural Science

Xiaofan Wang

University of Arkansas

\section{Samantha Howe}

University of Arkansas

\section{Hua Yang}

Zhejiang Academy of Agricultural Sciences

\section{Hua Li}

Foshan University

Jiangchao Zhao ( $\sim$ jzhao77@uark.edu )

University of Arkansas Fayetteville

\section{Ying Li}

Foshan University

\section{Research Article}

Keywords: Swine, Archaea, Energy Metabolism, CAZyme genes, ARGs

Posted Date: March 17th, 2021

DOI: https://doi.org/10.21203/rs.3.rs-305488/v1

License: (c) (i) This work is licensed under a Creative Commons Attribution 4.0 International License. Read Full License 


\section{Abstract \\ Background}

Archaea are an essential class of gut microorganisms in humans and animals. Despite the substantial progress in gut microbiome research in the last decade, most studies have focused on bacteria, and little is known about archaea in mammals. To this end, we investigated the composition, diversity, and functional potential of gut archaeal communities in pigs by reanalyzing a published metagenomic dataset from the NCBI Sequence Read Archive (SRA) database.

\section{Results}

A total of $276 \mathrm{fecal}$ samples from three countries: China $(n=76)$, Denmark $(n=100)$, and France $(n=100)$ were used in this study. For alpha diversity (Shannon Index) of the archaeal communities, Chinese pigs were less diverse than Danish and French pigs $(P<0.001)$. Consistently, Chinese pigs also possessed different archaeal community structures from the other two groups based on the Bray-Curtis distance matrix. Methanobrevibacter was the most dominant archaeal genus in Chinese pigs (44.94\%) and French pigs (15.41\%), while Candidatus Methanomethylophilus was the most predominant in Danish pigs (15.71\%). At the species level, the relative abundance of Candidatus Methanomethylophilus alvus, Natrialbaceae archaeon XQ INN 246, and Methanobrevibacter gottschalkii were greatest in Danish, French, and Chinese pigs with a relative abundance of $14.32 \%$, $11.67 \%$, and $16.28 \%$, respectively. In terms of metabolic potential, the top three pathways in the archaeal communities included the MetaCyc pathway related to the biosynthesis of L-valine, L-isoleucine, and isobutanol. Interestingly, the pathway related to hydrogen consumption (METHANOGENESIS-PWY) was only observed in archaeal reads, while the pathways participating in hydrogen production (FERMENTATION-PWY and PWY4LZ-257) were only detected in bacterial reads. Archaeal communities also possessed CAZyme gene families, with the top five being: AA3, GH43, GT2, AA6, and CE9. In terms of antibiotic resistance genes (ARGs), the class of multidrug resistance was the most abundant ARG, accounting for $87.41 \%$ of archaeal ARG hits.

\section{Conclusions}

Our study reveals the diverse composition and metabolic functions of archaea in pigs, suggesting that archaea might play important roles in swine nutrition and metabolism.

\section{Introduction}

Before Dr. Carl Woese and his colleagues separated archaea (named "Archaebacteria" at that time) from the bacteria domain based on phylogenetic analysis of ribosomal RNA sequences, archaea were considered a subgroup of bacteria[1]. To highlight the differences between archaea and bacteria, Dr. Carl Woese formally proposed the name of "Archaea" in his publication[2]. With the advancement of research technologies and tools, a large number of novel archaea and their characteristics have been discovered.

Archaea have highly diverse energy sources and unique metabolic characteristics and cell physiology, making it possible to live in various extreme environments, such as extreme temperature[3], high-salt[4], extreme alkaline[5], or acidic environments[6]. At the same time, archaea were also detected in various non-extreme ecosystems, including soil, ocean, lake water, and habitats associated with human and animal hosts, and were confirmed to make significant contributions to the ecological cycle by previous studies[7, 8]. As a "young" group with unique natural capabilities and biological characteristics, archaea have attracted increasing research interest in the past decade.

Unlike bacteria, archaea are one of the least studied and least understood members of the human microbiota. One of the reasons is that archaea only account for a very small proportion of the microbiota. A recent study among East Asians living in South Korea showed that archaea were detected in $42.47 \%$ (381/897) and constituted $10.24 \pm 4.58 \%$ of total bacteria and archaea among positive subjects[9]. However, the relatively small proportion of archaea does not mean it is not important. For 
example, some species of archaea have been reported to reduce Trimethylamine-N-oxide (TMAO), a harmful product associated with several diseases[10], through methanogenesis. In addition, methanogenic archaea can remove $\mathrm{H}_{2}$, the end product of bacterial fermentation, to increase the efficiency of fermentation and improve the energy harvesting capacity of the host[11]. Two studies performed on adults and children report that hydrogen-utilizing methanogenic archaea are co-enriched with $\mathrm{H} 2$ producing bacteria, Prevotella species, in the gut of obese humans[12]. Furthermore, human gut colonization by archaea has been associated with several other gastrointestinal and metabolic diseases, such as chronic constipation[13], inflammatory bowel disease[14], and colorectal cancer[15].

In pigs, the major ecological niches harboring archaea along the gastrointestinal tract (GIT) include the cecum, colon, and rectum, according to Gresse et al.[16]. The genus Methanobrevibacter is predominant with a relative abundance of 57-100\% of the archaeal communities in pigs and sows[16-19]. Additionally, the archaeal composition in the swine GIT is dynamic, according to Su et al. [17] and Federici et al.[20], and diet-driven [21]. These exploratory studies provided important information on archaea in pigs, however, their sample sizes were small, and the composition and metabolic potential of archaea at different production stages in pigs are still largely unknown.

Xiao et al. [22] established a swine gut microbiome reference gene catalog with metagenome deep sequencing of fecal samples from 287 pigs. In this study, we re-analyzed their data and investigated the diversity, composition, and potential functions of archaea in pigs.

\section{Materials And Methods}

\section{Obtaining data (Metadata (from Nature Microbiology))}

A total of $287 \mathrm{fecal}$ samples collected from China, France, and Denmark were sequenced using shotgun metagenomic sequencing technology by Xiao et al.[22], and we downloaded 276 of these sequences from the European Nucleotide Archive (ENA) under accession code PRJEB11755. We filtered 11 samples due to their low sequencing depths. In addition, we downloaded the predicted gene catalog generated by Liang et al. from GiGaDB (http://gigadb.org/dataset/view/id/100187/token/F4CDHYruxobOKmsE) to annotate the CAZy genes.

\section{Raw reads Pre-processing}

Downloaded raw reads were filtered using Kneaddata v0.7.2 (https://bitbucket.org/biobakery/kneaddata), an integrated pipeline used to perform quality control and remove host contamination from shotgun metagenomic data. In brief, reads were trimmed using Trimmomatic v0.39[23] with parameter SLIDINGWINDOW:4:20 and MINLEN:60. Potential host contamination was identified and removed by mapping all reads to the swine reference genome (Scrofa 11.1,

ftp://ftp.ncbi.nlm.nirefseq/vertebrate_mammalian/h.gov/genomes/Sus_scrofa/representative/GCF_000003025.6_Sscrofa11.1) using bmtagger v.3.102.4 [24] with default parameters. The number of clean metagenomic reads for fecal samples was rarefied to $16,230,988$ paired reads for each sample for a consistent sequencing depth by random subsampling.

\section{Taxonomic profiling and diversity}

Kraken2[25], a software used for short reads classification with the lowest common ancestor (LCA) algorithms, was used to classify clean short reads against the bacterial genome and archaeal genome databases. The bacterial genome database was downloaded using the Kraken2 standard command, which downloads NCBI taxonomic information and complete bacterial genomes in RefSeq (July 1, 2020)[26]. Then, clean reads were classified as bacterial taxa using Kraken2, and reads identified as bacteria were retained for downstream analysis. The method used for archaeal classification was slightly different compared with that of bacteria. The archaeal genome database was built using Kraken2 from two sources: RefSeq (July 1, 2020) and (2) a curated archaeal reference dataset[27]. Similarly, Kraken2 was used to assign clean reads into proper archaeal taxa based on this archaeal database, and archaeal reads were separated and retained.

Reads count table of archaea and bacteria at the species level were entered into QIIME2[28] platform to rarefy and calculate alpha diversity (Shannon Index) and beta diversity (Bray Curtis). 


\section{CAZyme and ARG analysis}

HUMAnN3[29] pipeline was used to characterize the metabolic pathways of archaea and bacteria using the MetaCyc database.

CAZyme family annotation was performed on the downloaded gene catalog generated by Xiao et al. via alignment with the dbCAN database[30] by HMMER3 software[31]. The genes identified as CAZy genes were extracted as target genes for further quantitative analysis. Salmon (quant)[32] was run on separated archaeal reads to quantify the archaeal CAZy genes in each sample, and a similar analysis was run on the separated bacterial reads.

Deeparg-SS[33] pipeline was adapted to predict antibiotic resistance genes in both bacterial and archaeal reads with the following critical parameters: -min-prob 0.8 -arg-alignment-identity 30 -arg-alignment-evalue $1 \mathrm{e}-10$.

\section{Statistical Analysis}

For all analyses, statistical significance was determined at $P<0.05$ and $P<0.001$. Kruskal-Wallis (pairwise) was performed to explore differences in alpha diversities among different countries. Analysis of similarities (ANOSIM) was carried out to test the statistical significance of beta diversity among different countries. Kruskal-Wallis (pairwise) and ANOSIM were run in QIIME2.

\section{Results}

\section{The diversity of archaea in pigs}

To assess the archaeal diversity in pigs, we re-analyzed 276 gut metagenomic sequences downloaded from Xiao et al. [22]. Significant differences in archaeal alpha diversity between different countries were observed. Pairwise comparisons of countries showed that archaeal diversity (Shannon Index) of Chinese pigs were significantly lower than that of the French (Fig. 1A, Kruskal-Wallis test, $P<0.001)$ and Danish groups $(P<0.001)$. No differences in the swine archaeal alpha diversity were observed between French and Danish $(P=0.918)$ pigs. Beta diversity of the archaeal communities was visualized on a principal coordinate analysis (PCoA) plot based on the Bray-Curtis distance matrix. Chinese pigs had significantly different archaeal communities from Danish (ANOSIM, $P<0.001, R=0.808$ ) and French (ANOSIM, $P<0.001, R=0.634$ ) pigs as demonstrated on the PCoA plot (Fig. 1B). However, the archaeal community structures of the Danish and French pigs could not be distinguished (Fig. 1B, ANOSIM, $P<0.001, \mathrm{R}=0.109$ ).

\section{The archaeal composition in pigs}

At the genus level, Methanobrevibacter, with an overall average relative abundance of $23.02 \%$, was the most abundant archaeal genus (Fig. 2), followed by Candidatus Methanomethylophilus, which had a relative abundance of $10.52 \%$. Of note, although Methanobrevibacter is considered the most dominant archaeal genus of pigs in general, it was ranked the second most abundant in Danish pigs. Additionally, Methanobrevibacter was significantly more abundant in Chinese pigs with the relative abundance of $44.94 \%$ than in both Danish (13.97\%) and French pigs (15.41\%), while Candidatus Methanomethylophilus was decreased in Chinese pigs (0.98\%) compared to Danish (15.71\%) and French (12.59\%) pigs.

At the species level, the mean relative abundance of the top 15 species is shown in Fig. 3. The gut microbiome of pigs is dominated by Natrialbaceae archaeon XQ INN 246 (mean relative abundance, 10.27\%), followed by Candidatus Methanomethylophilus alvus (9.57\%), Methanobrevibacter gottschalkii (6.40\%), and Methanobrevibacter smithii (3.42\%). The most abundant species in different countries seem to be inconsistent, as well. The relative abundance of Candidatus Methanomethylophilus alvus, Natrialbaceae archaeon XQ INN 246, and Methanobrevibacter gottschalkii were the highest in Danish, French, and Chinese pigs with an average relative abundance of $14.32 \%, 11.67 \%$, and $16.28 \%$, respectively.

\section{The metabolic pathways of the swine archaea}

To investigate the possible roles of gut archaea in swine, MetaCyc metabolic pathway profiles based on archaeal reads were investigated using HUMAnN3. A total of 77 MetaCyc pathways were observed, with 12 of these MetaCyc pathways present in 
more than $60 \%$ of fecal samples (Table S1). Figure 4A shows the top six most abundant MetaCyc pathways. The most abundant MetaCyc pathway was involved in pyruvate fermentation to isobutanol (PWY-7111). Pathways of VALSYN-PWY and ILEUSYN-PWY ranked as second and third and were related to the biosynthesis of two essential amino acids, L-valine and Lisoleucine, respectively. Interestingly, these pathways, PWY-7111, VALSYN-PWY, and ILEUSYN-PWY, were also among the top 10 most abundant MetaCyc pathways in bacteria (Fig. 4B, Table S2). Furthermore, the pathway related to hydrogen consumption (METHANOGENESIS-PWY) was only observed in archaea and not in bacteria (Table S1 and Table S2). In contrast, the pathways involved in hydrogen production (FERMENTATION-PWY and PWY4LZ-257) were only observed in bacteria and not in archaea (Fig. 5).

We next profiled the archaea-annotated CAZyme gene families in the swine gut. A total of 297 CAZyme gene families were identified from archaeal reads, out of which 99 were detected with a sample coverage of more than $60 \%$. The three major CAZyme families were glycoside hydrolases (GH, 54/99), glycoside transferases (GT, 24/99), and carboxylesterase (CE, 11/99). The most abundant CAZyme category was AA3, followed by GH43 and GT2 (Fig. 6A). For comparison, we also investigated bacterial CAZyme profiles (Fig. 6B) and found that six of the top 10 CAZyme families of archaea and bacteria were shared, including $A A 3, G T 2, A A 6, G T 41, G T 5$, and GH13.

Finally, we examined the ARGs in the swine gut archaea. In total, 13 classes of ARGs were detected from archaeal reads in all samples. On average, $0.035 \%$ of ARG hits were obtained from archaeal reads. The ARG class of multidrug resistance represented the vast majority (87.41\%) of ARG hits. The other ARG classes with more than $1 \%$ relative abundance include peptide (5.07\%), tetracycline (2.29\%), mupirocin (1.69\%), and unclassified (1.15\%). The ARG type of multidrug class was significant enriched in Chinese pigs (Kruskal-Wallis test, $P<0.01$ ), and the relative abundance of the multidrug class in Danish pigs is significantly higher than that in French pigs (Kruskal-Wallis test, $P<0.01$ ). The boxplots of the top six ARG genes are shown in Fig. 6C. In contrast, 24 ARGs were identified in bacterial reads, and $0.144 \%$ of bacterial reads were identified as ARG hits. The most abundant ARG class in bacteria was tetracycline, accounting for $40.59 \%$ of the total ARG hits (Fig. 6D), while the class of multidrug ranked second with $19.39 \%$, followed by MLS (17.73\%), beta-lactam (7.46\%), peptide (5.33\%), and aminoglycoside (4.63\%).

\section{Discussion}

Although archaea are an essential class of gut microorganisms in humans and animals, they have been neglected for many years. A few studies have examined archaeal composition by 16S rRNA sequencing methods; however, most studies only focused on the composition of methanogenic archaea of gut archaea. Furthermore, only a small number of gut archaeal strains were isolated from pig feces[34]. Therefore, compared to bacteria, very limited information is available for the archaeal composition in pigs, and even less is known about the archaeal metabolic pathways in pigs. In this study, by re-analyzing the swine gut metagenome of a large number of pigs from three countries, we characterized the swine gut archaeal diversity and metabolic pathways, which improves our understanding of the potential roles of archaea in mammals.

Regarding archaeal diversity, Chinese pigs had archaeal communities significantly different from Danish and French pigs in both alpha and beta diversity, consistent with the bacterial communities described by Xiao and colleagues[22]. Chinese pigs were fed two types of antibiotics, including fluorine benzene $(400 \mathrm{~g} /$ ton) and penicillin $(600 \mathrm{~g} /$ ton $)$, which may cause a significant reduction in microbial richness. However, most archaea were not sensitive to penicillin and fluorine benzene because of the absence of drug targets[35], which means that the administration of antibiotics could not fully explain the decrease of archaeal richness in Chinese pigs. Geography, environment, diet, sex, and breed are confounding factors that might contribute to the difference in alpha and beta diversity of the swine gut archaeal diversity between the three countries.

As for archaeal composition, Methanobrevibacter was the most abundant archaeal genus in general, which was in line with previous studies[16]. It should be noted that variations in the abundance of Methanobrevibacter between countries were observed. It was ranked the second most abundant genus in the Danish group with a relative abundance of $13.97 \%$, following Candidatus Methanomethylophilus (relative abundance of 15.71\%). The archaeal composition based on metagenomics was different from that revealed by 16 s rRNA or mcrA genes. Mi and colleagues found that the genus Methanobrevibacter was the 
dominant archaeal taxon with an average relative abundance of $57 \%$ in the large intestine of finishing pigs[19]. Furthermore, Methanobrevibacter was also reported as the overwhelmingly dominant archaeal genus and accounted for $95.01-100 \%$ of all archaeal reads in pigs in other studies[17, 18]. Many factors, such as environment, diet, breed, and age, might contribute to the discrepancies in the archaeal composition between these studies. It is worth noting that technology (i.e., shotgun metagenomics sequencing vs. 16S rRNA sequencing) is likely another important confounder. Amplification bias is an inevitable disadvantage to accurately quantify bacterial and archaeal cells[36]. Of particular note, compared with bacteria, the domain archaea remains poorly understood, and there lacks enough reference sequences to design primers properly, which causes many archaeal taxa to remain undetected[37].

Previous studies on animals and humans have revealed that archaea were involved in energy metabolism through the consumption of hydrogen and carbon dioxide to produce methane[11,38]. In our study, the metabolic pathway related to methanogenesis from $\mathrm{H}_{2}$ and $\mathrm{CO}_{2}$ (METHANOGENESIS-PWY) was only found in archaeal reads and not in bacterial reads. In contrast, hydrogen production pathways were only found in bacterial reads and not in archaeal reads. These results suggest that there is a complementary relationship between archaea and bacteria, and archaea participated in energy metabolism in pigs as well. Samuel and colleagues showed that methanogenic archaea were able to enhance caloric harvest and weight gain by methods other than hydrogen-utilization in humans, but the detailed mechanism is unclear[38]. We inferred that archaea have the potential to improve the growth performance of pigs through the synthesis of essential amino acids. In our study, Lvaline and L-isoleucine -related pathways were ranked as the top MetaCyc pathways of archaea. L-valine and L-isoleucine are essential amino acids and could improve the growth performance of pigs at multiple stages of growth[39, 40]. Of note, the two pathways were also abundant in bacteria. Further studies are desired to elucidate the proportion and magnitude of archaeal contribution to amino acid biosynthesis in pigs.

Interestingly, a large number of CAZyme gene families were also detected in archaeal reads. CAZyme genes are involved in carbohydrate metabolism and are important in the absorption and utilization efficiency of energy for the host. CAZyme gene families abundance in the host gut were confirmed association with growth performance in several domestic animals [41-43]. In general, bacteria in the mammal gut, especially species belonging to Firmicutes and Bacteroidetes, were considered the main providers of CAZyme genes for encoding enzymes to digest complex carbohydrates[44]. Nevertheless, recent publications revealed that archaea participate in carbohydrate metabolism through the production of various enzymes[45, 46]. In our study, 297 CAZyme gene families were detected in archaea reads, and six of the top 10 CAZyme families of archaea and bacteria were shared. These CAZyme gene families of archaea may be useful supplements for carbohydrate digestion in swine. Therefore, our study suggests that, in addition to $\mathrm{H}_{2}$ depletion, archaea might be directly involved in energy metabolism in the pig gut.

Bacterial ARGs have been investigated in many studies in different animals. However, little is known about ARGs in archaea. In this study, we also explored the archaeal ARGs in pigs in addition to metabolic pathways. The ARG classes of tetracycline, multidrug, and MLS were relatively abundant in bacterial reads of the 287 pigs. However, the ARG class of multidrug resistance represented the overwhelming majority at $87.41 \%$ of ARG hits in archaeal reads of these pigs. The effects of low-dose antibiotics on archaeal ARGs in pigs, the generation and spread of ARGs among archaeal and between domains need further investigation.

In this study, we profiled archaeal composition and functional potential in pigs. Although amplification bias was avoided by using shotgun metagenomics data, there are two limitations of our study. First, our study only included a limited number of archaeal reference genomes. Therefore, numerous archaea with no reference genomes could not be identified, which can underestimate the richness of archaea and its functional diversity. Second, compared with bacteria, the relative abundance of archaea is lower by about $1 \%$. Therefore, higher sequencing depth was demanded to accurately quantify archaea.

Although we demonstrated archaea had functional potentials, further studies are needed to link archaeal species or strains with growth performance of pigs. In addition, methane, the production of methanogenic archaea, is a greenhouse gas with environmental concerns. Thus, modulation of archaeal communities to maintain a balance between animal production (e.g., growth-promoting functions) and environmental protection (e.g., methane emission) is critical. 


\section{Conclusion}

In conclusion, our study revealed the composition and functional potential of archaea in the swine gut based on the re-analysis of metagenomic data. We have demonstrated different predominant archaeal genera and species in different swine populations. In terms of functional potential, METHANOGENESIS-PWY related to methanogenesis from $\mathrm{H}_{2}$ and $\mathrm{CO}_{2}$ was only found in archaeal reads, which indicated that archaea were involved in energy metabolism by depleting $\mathrm{H}_{2}$ in the swine gut. Moreover, archaea shared several amino acid synthesis pathways and the main CAZyme gene families with bacteria, which suggested archaea are potentially involved in amino acid synthesis and energy metabolism.

\section{Abbreviations}

ARGs: Antibiotic resistant genes; CAZyme: Carbohydrate-active enzyme; TMAO: Trimethylamine-N-oxide; NCBI: National Center for Biotechnology Information; PCOA: Principal coordinate analysis; ANOSIM: Analysis of similarity.

\section{Declarations}

\section{Ethics approval and consent to participate}

Not applicable.

\section{Consent for publication}

Not applicable.

\section{Availability of data and materials}

Not applicable.

\section{Competing of Interests}

The authors declare that they have no competing interests.

\section{Authorship contribution}

Study and experiments were conceived and designed by JZ and YL. Resource, methodology and analysis tools were contributed by $F L, Y L, X W, Y X, X W$ and FD. Data analysis and interpretation were performed by FD, HL, HY and JZ. The manuscript was written and prepared by FD, YL and JZ. All authors read and approved the final manuscript.

\section{Funding}

This work was supported by the Guangdong Provincial Key Laboratory of Animal Molecular Design and Precise Breeding (2019B030301010), and the Key Laboratory of Animal Molecular Design and Precise Breeding of Guangdong Higher Education Institutes (2019KSYS011).

\section{Acknowledgments}

Computational support was provided by the Extreme Science and Engineering Discovery Environment (XSEDE)[47], which is supported by National Science Foundation grant number ACl-1548562. Specifically, it used the Bridges-2 system, which is supported by NSF award number ACl-1445606, at the Pittsburgh Supercomputing Center (PSC).

\section{References}

1. Woese CR, Fox GE. Phylogenetic structure of the prokaryotic domain: the primary kingdoms. PNAS. 1977;74:5088-90. 
2. Woese CR, Kandler O, Wheelis ML. Towards a natural system of organisms: proposal for the domains Archaea, Bacteria, and Eucarya. PNAS. 1990;87:4576-9.

3. Cavicchioli R. Cold-adapted archaea. Nat Rev Microbiol. 2006;4:331-43.

4. Andrei A-Ş, Banciu HL, Oren A. Living with salt: metabolic and phylogenetic diversity of archaea inhabiting saline ecosystems. FEMS Microbiol Lett. 2012;330:1-9.

5. Shen Jp, Zhang Lm, Zhu Yg, Zhang Jb, He Jz. Abundance and composition of ammonia-oxidizing bacteria and ammoniaoxidizing archaea communities of an alkaline sandy loam. Environ Microbiol. 2008;10:1601-11.

6. Herbold CW, Lehtovirta-Morley LE, Jung MY, Jehmlich N, Hausmann B, Han P, et al. Ammonia-oxidising archaea living at low pH: Insights from comparative genomics. Environ Microbiol. 2017;19:4939-52.

7. Moissl-Eichinger C, Pausan M, Taffner J, Berg G, Bang C, Schmitz RA. Archaea are interactive components of complex microbiomes. Trends in microbiology. 2018;26:70-85.

8. Borrel G, Brugere J-F, Gribaldo S, Schmitz RA, Moissl-Eichinger C. The host-associated archaeome. Nat Rev Microbiol. 2020;18:622-36.

9. Kim JY, Whon TW, Lim MY, Kim YB, Kim N, Kwon M-S, et al. The human gut archaeome: identification of diverse haloarchaea in Korean subjects. Microbiome. 2020;8:114.

10. Bain MA, Faull R, Fornasini G, Milne RW, Evans AM. Accumulation of trimethylamine and trimethylamine-N-oxide in endstage renal disease patients undergoing haemodialysis. Nephrol Dial Transplant. 2006;21:1300-4.

11. Turnbaugh PJ, Ley RE, Mahowald MA, Magrini V, Mardis ER, Gordon JI. An obesity-associated gut microbiome with increased capacity for energy harvest. nature. 2006;444:1027.

12. Zhang H, DiBaise JK, Zuccolo A, Kudrna D, Braidotti M, Yu Y, et al. Human gut microbiota in obesity and after gastric bypass. PNAS. 2009;106:2365-70.

13. Pimentel M, Gunsalus RP, Rao SS, Zhang H. Methanogens in human health and disease. Am J Gastroenterol Suppl. 2012;1:28.

14. Khelaifia S, Raoult D. Haloferax massiliensis sp. nov., the first human-associated halophilic archaea. New Microbes New Infect. 2016;12:96.

15. Coker OO, Wu WKK, Wong SH, Sung JJ, Yu J. Altered gut archaea composition and interaction with bacteria are associated with colorectal cancer. Gastroenterology. 2020;159:1459-70. e5.

16. Gresse R, Chaucheyras Durand F, Dunière L, Blanquet-Diot S, Forano E. Microbiota composition and functional profiling throughout the gastrointestinal tract of commercial weaning piglets. Microorganisms. 2019;7:343.

17. Su Y, Bian G, Zhu Z, Smidt H, Zhu W. Early methanogenic colonisation in the faeces of Meishan and Yorkshire piglets as determined by pyrosequencing analysis. Archaea. 2014;2014.

18. Luo Y-h, Su Y, Wright A-DG, Zhang L-I, Smidt H, Zhu W-y. Lean breed Landrace pigs harbor fecal methanogens at higher diversity and density than obese breed Erhualian pigs. Archaea. 2012;2012:605289.

19. Mi J, Peng H, Wu Y, Wang Y, Liao X. Diversity and community of methanogens in the large intestine of finishing pigs. $B M C$ Microbiol. 2019;19:83.

20. Federici S, Miragoli F, Pisacane V, Rebecchi A, Morelli L, Callegari ML. Archaeal microbiota population in piglet feces shifts in response to weaning: Methanobrevibacter smithii is replaced with Methanobrevibacter boviskoreani. FEMS Microbiol Lett. 2015;362:fnv064.

21. Luo Y, Chen H, Yu B, He J, Zheng P, Mao X, et al. Dietary pea fiber increases diversity of colonic methanogens of pigs with a shift from Methanobrevibacter to Methanomassiliicoccus-like genus and change in numbers of three hydrogenotrophs. BMC Microbiol. 2017;17:17.

22. Xiao L, Estellé J, Kiilerich P, Ramayo-Caldas Y, Xia Z, Feng Q, et al. A reference gene catalogue of the pig gut microbiome. Nat Microbiol. 2016;1:16161.

23. Bolger AM, Lohse M, Usadel B. Trimmomatic: a flexible trimmer for Illumina sequence data. Bioinformatics. 2014;30:211420. 
24. Rotmistrovsky K, Agarwala R. BMTagger: Best Match Tagger for removing human reads from metagenomics datasets. Unpublished. 2011.

25. Wood DE, Lu J, Langmead B. Improved metagenomic analysis with Kraken 2. Genome biology. 2019;20:257.

26. Haft DH, DiCuccio M, Badretdin A, Brover V, Chetvernin V, O'Neill K, et al. RefSeq: an update on prokaryotic genome annotation and curation. Nucleic Acids Res. 2018;46:D851-D60.

27. Kirstahler P, Bjerrum SS, Friis-Møller A, La Cour M, Aarestrup FM, Westh H, et al. Genomics-based identification of microorganisms in human ocular body fluid. Sci Rep. 2018;8:1-14.

28. Bolyen E, Rideout JR, Dillon MR, Bokulich NA, Abnet CC, Al-Ghalith GA, et al. Reproducible, interactive, scalable and extensible microbiome data science using QIIME 2. Nat Biotechnol. 2019;37:852-7.

29. Franzosa EA, Mclver LJ, Rahnavard G, Thompson LR, Schirmer M, Weingart G, et al. Species-level functional profiling of metagenomes and metatranscriptomes. Nat Methods. 2018;15:962-8.

30. Zhang H, Yohe T, Huang L, Entwistle S, Wu P, Yang Z, et al. dbCAN2: a meta server for automated carbohydrate-active enzyme annotation. Nucleic Acids Res. 2018;46:W95-W101.

31. Eddy SR: A new generation of homology search tools based on probabilistic inference. In: Genome Informatics 2009: Genome Informatics Series Vol. 23. World Scientific; 2009: 205-11.

32. Patro R, Duggal G, Love MI, Irizarry RA, Kingsford C. Salmon provides fast and bias-aware quantification of transcript expression. Nat Methods. 2017;14:417-9.

33. Arango-Argoty G, Garner E, Pruden A, Heath LS, Vikesland P, Zhang L. DeepARG: a deep learning approach for predicting antibiotic resistance genes from metagenomic data. Microbiome. 2018;6:1-15.

34. Miller TL, Wolin M, Kusel E. Isolation and characterization of methanogens from animal feces. Syst App/ Microbiol. 1986;8:234-8.

35. Al-Mailem D, Sorkhoh N, Al-Awadhi H, Eliyas M, Radwan S. Biodegradation of crude oil and pure hydrocarbons by extreme halophilic archaea from hypersaline coasts of the Arabian Gulf. Extremophiles. 2010;14:321-8.

36. Kennedy K, Hall MW, Lynch MD, Moreno-Hagelsieb G, Neufeld JD. Evaluating bias of Illumina-based bacterial 16S rRNA gene profiles. Applied and environmental microbiology. 2014;80:5717-22.

37. Eloe-Fadrosh EA, Ivanova NN, Woyke T, Kyrpides NC. Metagenomics uncovers gaps in amplicon-based detection of microbial diversity. Nat Microbiol. 2016;1:1-4.

38. Samuel BS, Gordon JI. A humanized gnotobiotic mouse model of host-archaeal-bacterial mutualism. PNAS. 2006;103:10011-6.

39. Mavromichalis I, Kerr B, Parr T, Albin D, Gabert V, Baker D. Valine requirement of nursery pigs. Anim Sci J. 2001;79:1223-9.

40. Figueroa J, Lewis A, Miller P, Fischer R, Diedrichsen R. Growth, carcass traits, and plasma amino acid concentrations of gilts fed low-protein diets supplemented with amino acids including histidine, isoleucine, and valine. Anim Sci J. 2003;81:152937.

41. Alfaia C, Pestana J, Rodrigues M, Coelho D, Aires M, Ribeiro D, et al. Influence of dietary Chlorella vulgaris and carbohydrate-active enzymes on growth performance, meat quality and lipid composition of broiler chickens. Poult Sci. 2020.

42. Jiang B, Wang T, Zhou Y, Li F. Effects of enzyme+ bacteria treatment on growth performance, rumen bacterial diversity, KEGG pathways, and the CAZy spectrum of Tan sheep. Bioengineered. 2020;11:1221-32.

43. Fang S, Chen X, Ye X, Zhou L, Xue S, Gan Q. Effects of gut microbiome and short-chain fatty acids (SCFAs) on finishing weight of meat rabbits. Front Microbiol. 2020;11:1835.

44. White BA, Lamed R, Bayer EA, Flint HJ. Biomass utilization by gut microbiomes. Annu Rev Microbiol. 2014;68:279-96.

45. Kırtel O, Lescrinier E, Van den Ende W, Öner ET. Discovery of fructans in Archaea. Carbohydr Polym. 2019;220:149-56.

46. Suleiman M, Krüger A, Antranikian G. Biomass-degrading glycoside hydrolases of archaeal origin. Biotechnol Biofuels. 2020;13:1-14. 
47. Towns J, Cockerill T, Dahan M, Foster I, Gaither K, Grimshaw A, et al. XSEDE: accelerating scientific discovery. Comput Sci Eng. 2014;16:62-74.

\section{Figures}
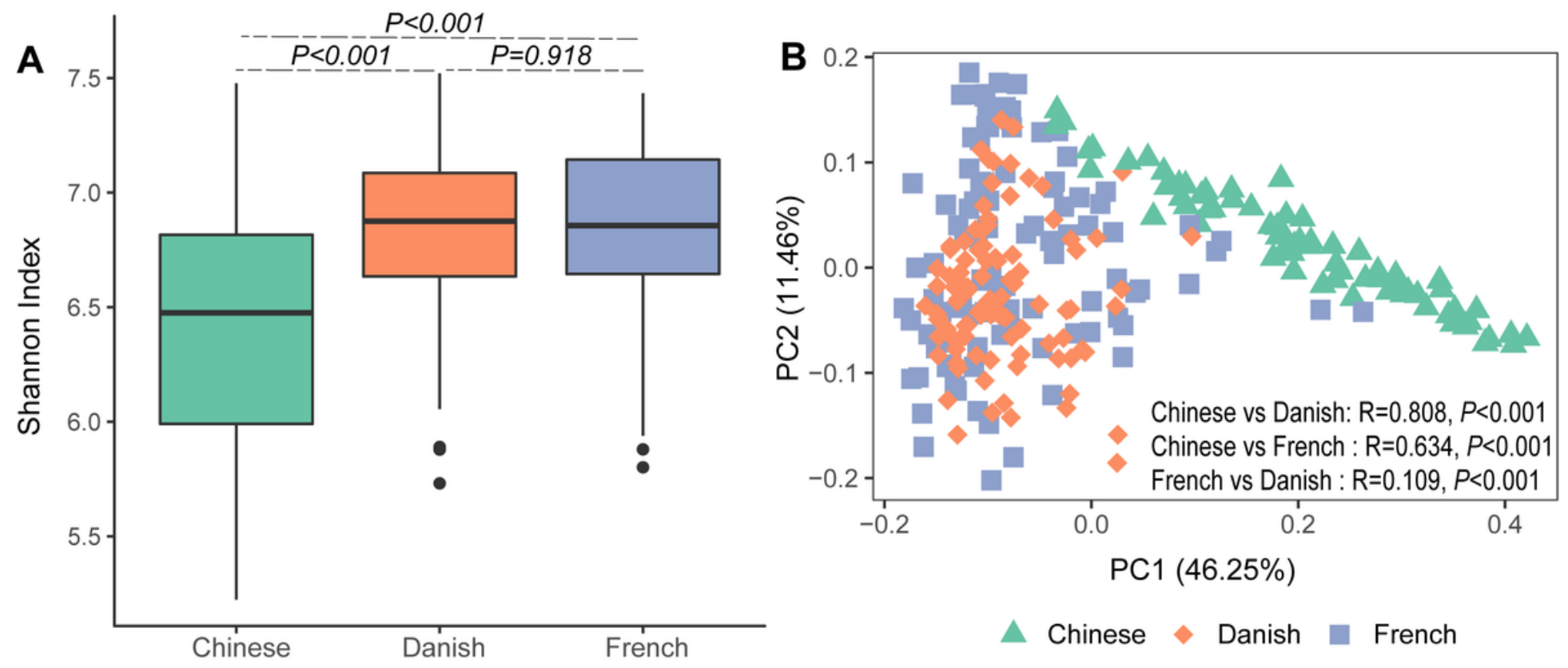

\section{Figure 1}

Swine archaeal alpha and beta diversities between three countries. Alpha diversity of archaeal communities in pigs was measured using Shannon Index (A). Beta diversity was illustrated on principal coordinate analysis (PCoA) plot based on BrayCurtis distance (B).
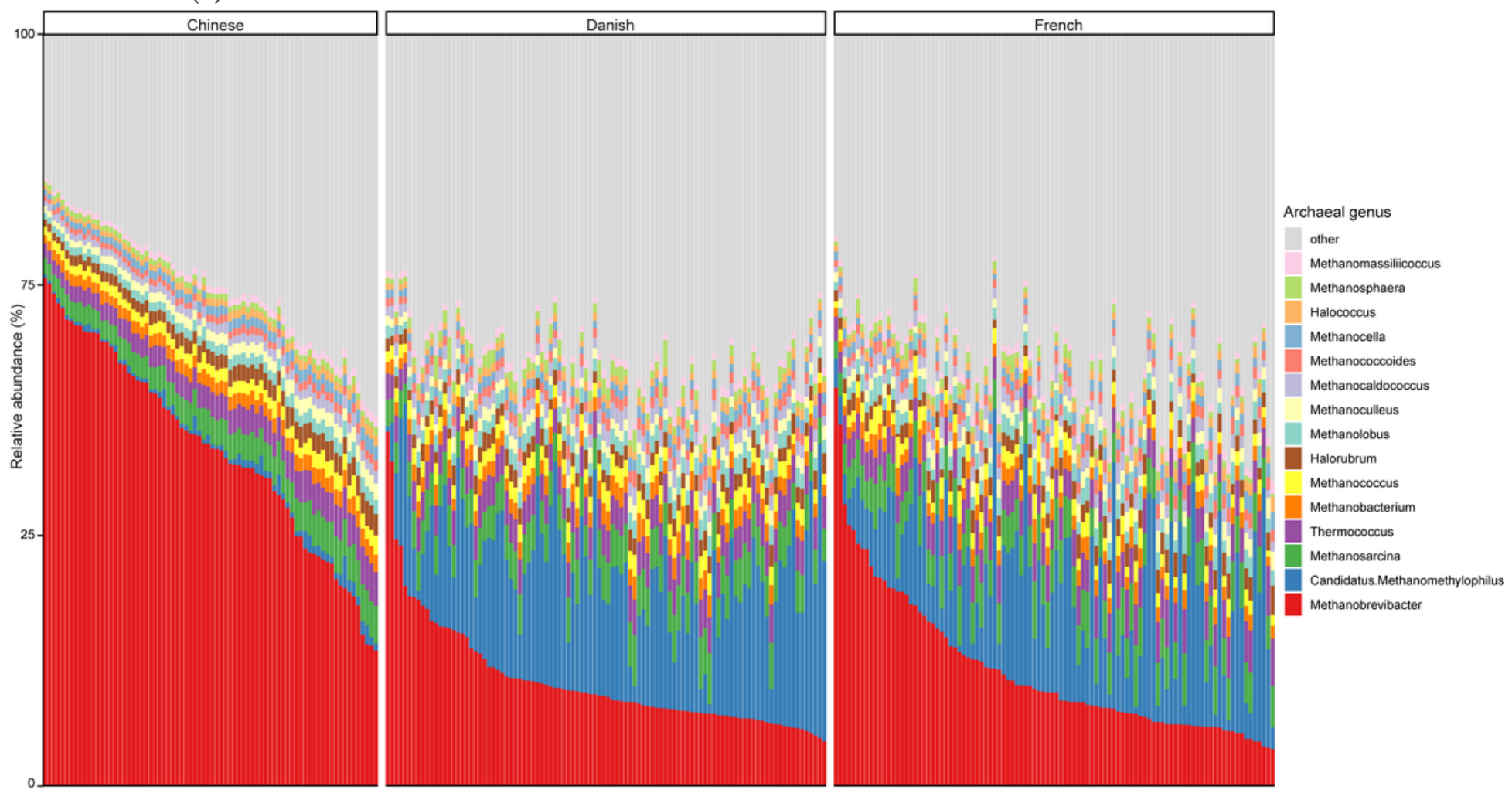

Figure 2 
Relative abundance of the top 15 archaeal genera in swine fecal samples from different countries. Each column represents one archaeal community, and each color represents one archaeal genus. The y-axis represents relative abundance ranging from 0 to $100 \%$.
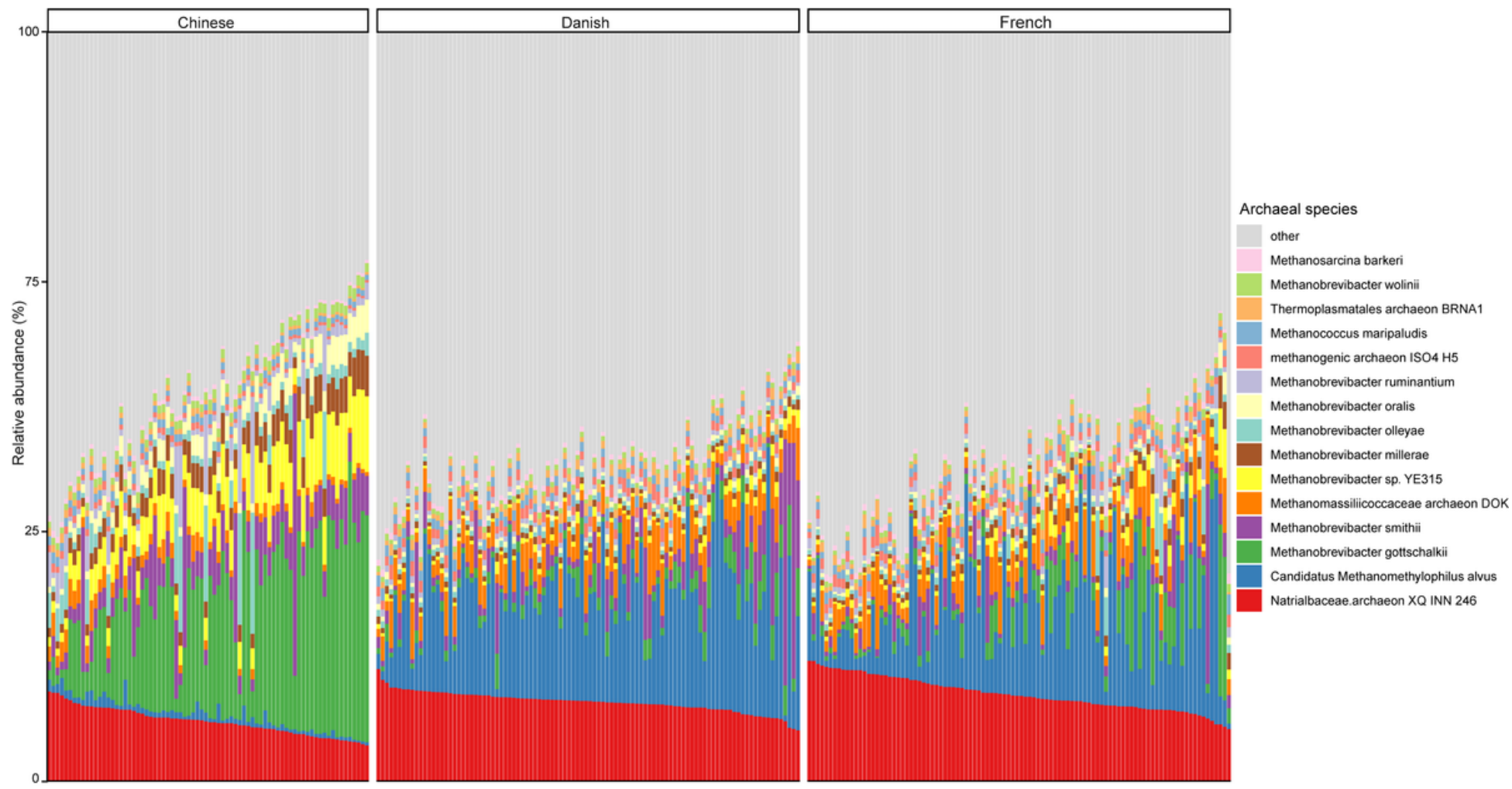

Figure 3

Relative abundance of the top 15 archaeal species in swine fecal samples from different countries. Each column represents one archaeal community, and each color represents one archaeal species. The $y$-axis represents relative abundance ranging from 0 to $100 \%$.
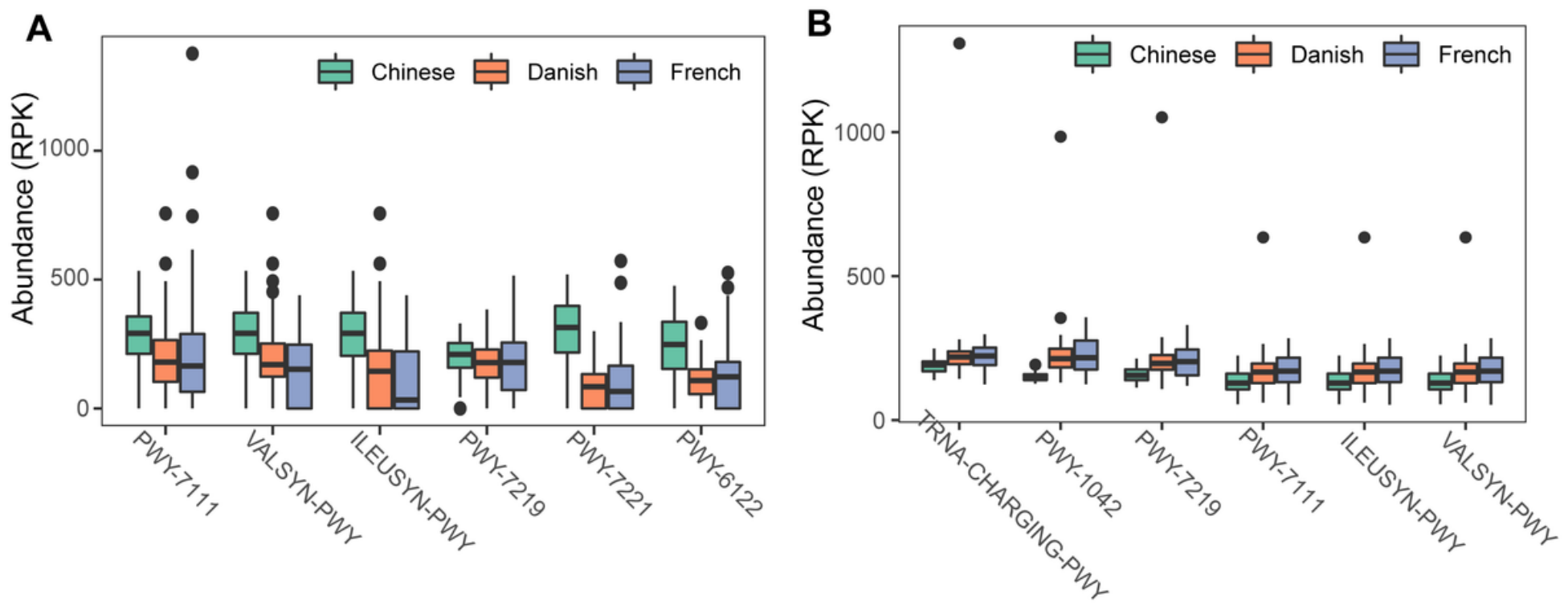

\section{Figure 4}

Summary of the predominant MetaCyc pathways of archaea and bacteria in pigs. The top six most abundant MetaCyc pathways of archaea (A) and bacteria (B) are displayed. In A and B, the $y$-axis represents the number of reads mapped to 
reference genes involved in pathways. Data have been normalized to RPKM (reads per kilobase of transcript per million mapped reads).

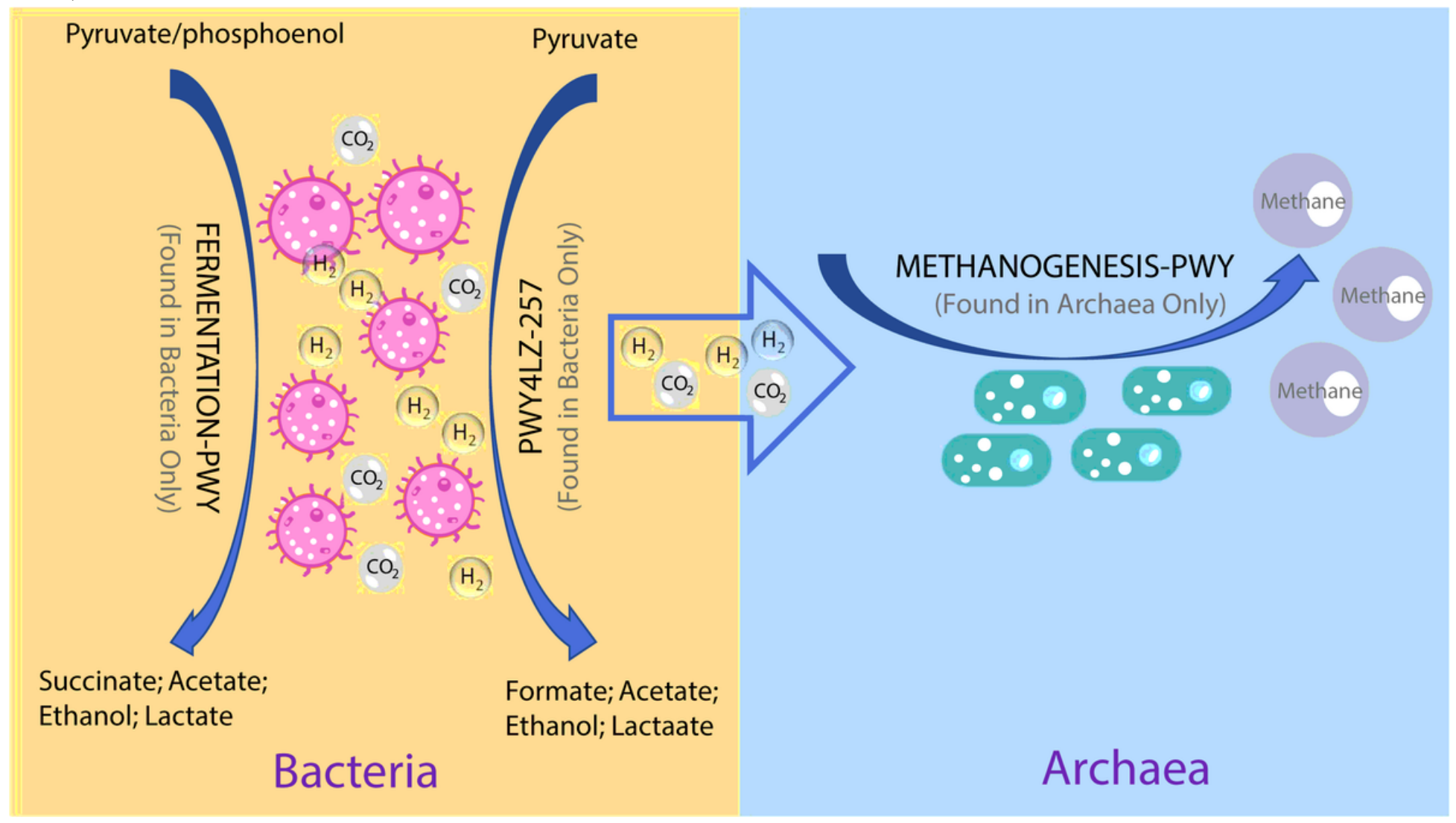

\section{Figure 5}

Overview of the hydrogen production pathways of bacteria and consumption pathway of archaea. 
A

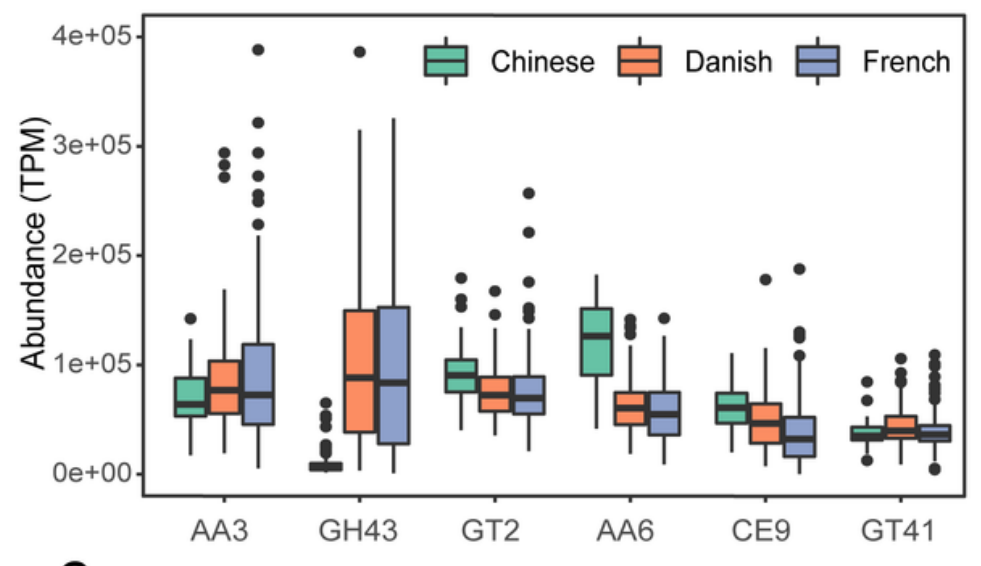

C

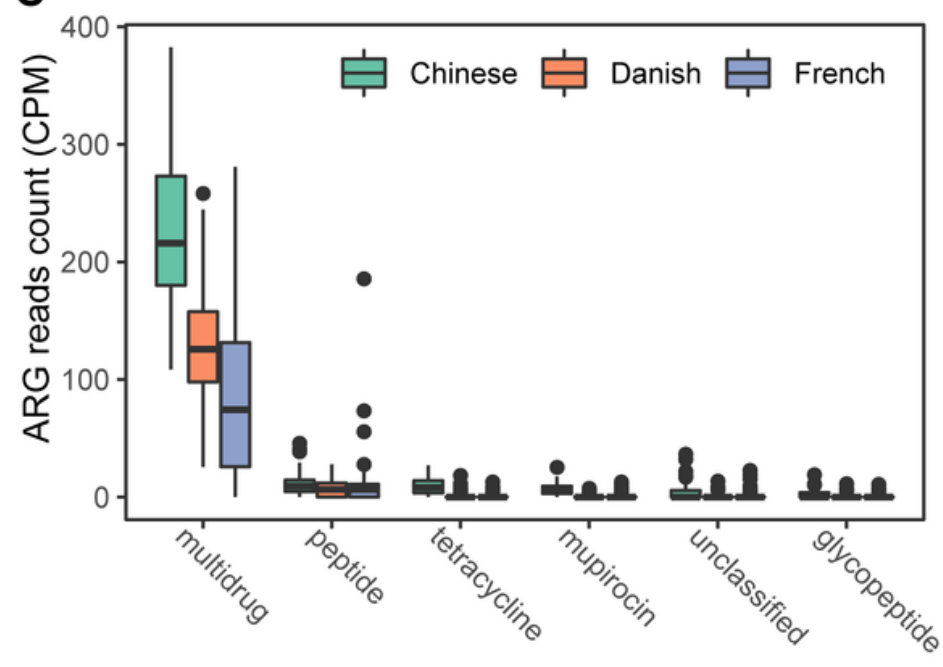

B

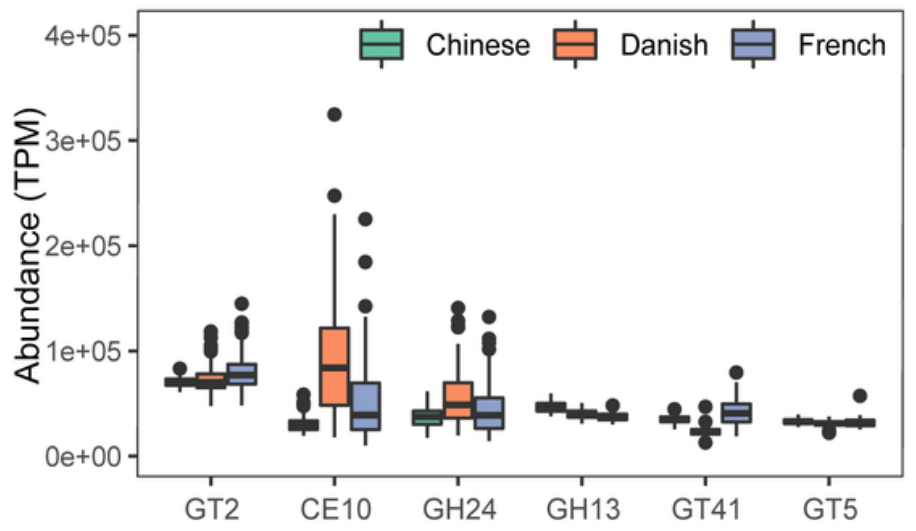

D

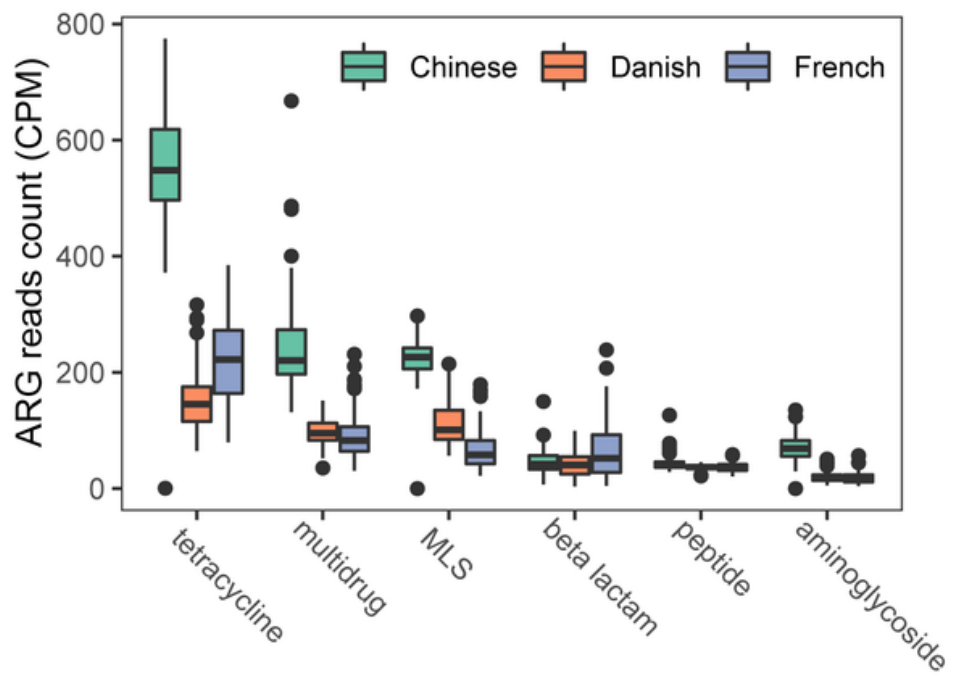

Figure 6

Summary of predominant CAZyme gene families (A and B) and antibiotic resistance genes (C and D) of archaea and bacteria in pigs. Top six CAZy gene families of archaea (A) and bacteria (B) were normalized by TPM (Trans Per Million) using salmon software. ARG raw counts were identified using deeparg software. The ARG reads count of archaea (C) and bacteria (D) were normalized by CPM (Counts Per Million).

\section{Supplementary Files}

This is a list of supplementary files associated with this preprint. Click to download.

- Suppmentary.rar 